\title{
Diagnóstico de la organización funcional del sistema urbano en los planes de ordenamiento territorial departamental: el caso de Córdoba -Síntesis Metodológica -
}

Franz Gutiérrez Rey*

Resumen. El presente artículo muestra algunos de los resultados más relevantes del diagnóstico de la organización funcional del sistema urbano del departamento de Córdoba, obtenidos a través de la metodología propuesta por el autor para realizar este tipo de investigación aplicada, en el marco de las políticas actuales de Ordenamiento Territorial -OT (Gutiérrez, 2001).

El diagnóstico de la organización funcional del sistema urbano departamental -DOFSUD-, aporta los aspectos básicos necesarios para el Plan de Ordenamiento Territorial Departamental -POTD-y contribuye a que las autoridades departamentales y municipales dispongan de áreas o subregiones funcionales, las cuales constituyen la síntesis espacial del territorio departamental para el POTD, al reflejar las condiciones de todos los componentes territoriales -económicos, ambientales, sociales y políticosy representar el equilibrio de éstos, por lo que se establecen como el soporte espacial "ideal" para la acción, aplicación y concreción de las políticas de planificación y de ordenamiento territorial en el departamento. Palabras clave: jerarquía urbana, centro urbano, región funcional, función, servicios, equipamiento, rango, tamaño, relaciones, vínculos, flujos, volumen, área de atracción o influencia, nodo, polo, red, malla, sistema, organización.

\footnotetext{
Geógrafo; Licenciado con Estudios Mayores en Geografía y Básicos en Socioeconomía; especializado en Computación para la Docencia e Interpretación de Imágenes de Sensores Remotos Aplicada a Levantamientos Rurales; Magíster en Geografía con Énfasis en Ordenamiento Territorial. franz@geordena.com - http://www.geordena.com
} 


\section{Introducción}

El análisis de la organización funcional de los sistemas urbanos regionales y subregionales hace parte de los componentes de diagnóstico del OTD y fortalece el criterio en el país de lo imprescindible que es la Geografía en los procesos de planificación y más si se trata de desconcentrar el desarrollo, la riqueza, las oportunidades, es decir el bienestar en el territorio.

El diagnóstico de la organización funcional del sistema urbano -DOFSU- para el OTD, permite a los planificadores del departamento un mayor equilibrio espacial en: la inversión pública, las redes de transporte, la localización o distribución de los servicios, equipamientos sociales, económicos y administrativos esenciales y la desconcentración o concentración espacial de las actividades económicas, administrativas, de servicios y equipamientos. Así mismo, en la disminución o potencialización del crecimiento físico de los centros, la estimulación o no de movimientos migratorios hacia los centros urbanos, el fortalecimiento de las redes de mercado, la integración de los centros urbanos a través de la presencia de instituciones del estado y de infraestructura de comunicaciones para la producción; para de esta manera garantizar la presencia del Estado con alternativas de solución espaciales a problemas articulados en el contexto integral y sistémico del departamento.

Los procedimientos metodológicos establecidos y desarrollados para el DOFSUD avalan su fácil adaptación y aplicación a las situaciones particulares de cada uno de los departamentos del país por su: bajo costo, utilización de técnicas y herramientas de investigación cómodas de seguir -entendibles por quienes diseñan las políticas, así posean poca o ninguna experiencia en procesos de planificación, desarrollo u ordenamiento, no dependencia de software y equipos sofisticados -SIG-, posibilidad de realizarse con poco personal, en poco tiempo y con datos exclusivamente de las oficinas de planeación departamental y por que sus resultados pueden ser incorporados rápidamente a los Planes de Ordenamiento Territorial Municipal POTM- o departamentales -POTD-, siendo confiables y objetivos ${ }^{1}$, pese a su simplicidad.

El DOFSUD, se desarrolla a partir del análisis y síntesis de cuatro análisis claves para el OTD, denominados procesos: urbanos, espaciales, de subregionalización $\mathrm{y}$ de regiones funcionales (Figura 1).

\section{Los procesos urbanos}

Son analizados a través de la jerarquía, morfología, funciones y dinámicas urbanas presentes en el departamento (Figura 1).

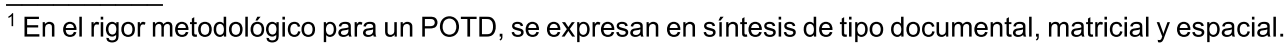

40 Franz Gutiérrez Rey 


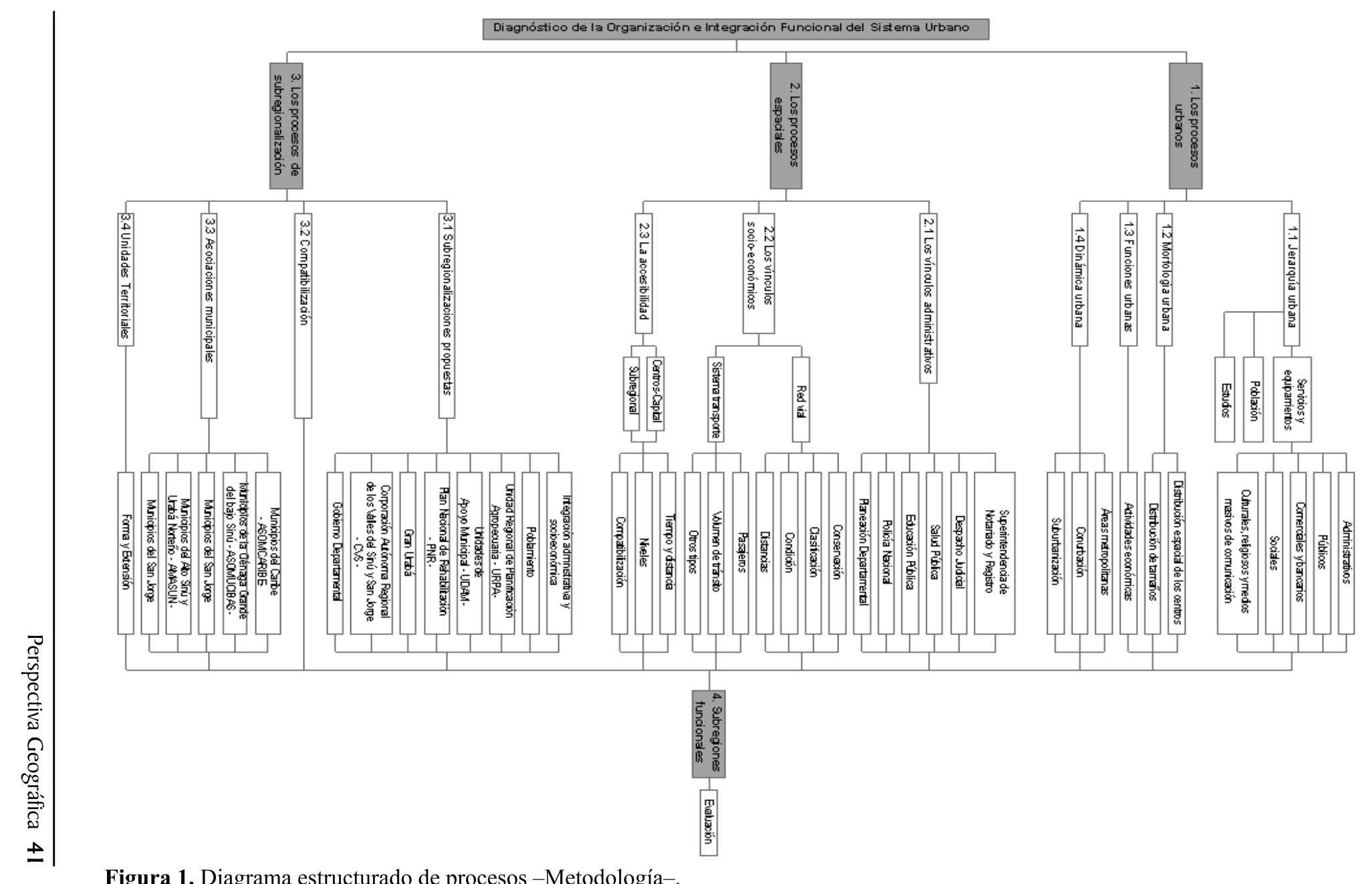




\subsection{La jerarquía urbana}

Se establece inicialmente, por la oferta de servicios y equipamientos -la mayor o menor concentración de servicios y equipamientos en los centros urbanos de un departamento, hace posible su clasificación jerárquica, por que de la oferta de éstos depende el nivel de importancia e influencia-, identificándose cinco componentes de servicios y equipamientos en el departamento: administrativos, públicos básicos, comerciales y bancarios, sociales y culturales. Cada componente es desarrollado a través de variables que representan los servicios y equipamientos con que cuenta cada centro urbano y estas, por medio de indicadores de rango o clase (Tabla 1).

Recopilada la información en matrices y ponderadas en orden decreciente para cada uno de los centros urbanos del departamento, se establecen grupos por análisis simple de oferta -sumatoria- y estadístico -frecuencias-, los cuales corresponden a los distintos niveles jerárquicos de los centros urbanos por primacía en la oferta de servicios y equipamientos (Figura 2).

A continuación, la clasificación jerárquica por servicios y equipamientos es validada por el análisis de la jerarquía urbana por tamaño de la población -a mayor población es mayor la oferta de servicios y equipamientos, por ende el nivel en la jerarquía urbana y viceversa-, que junto con el análisis de la dinámica de la población es complementado, obteniéndose una mayor seguridad en la clasificación de los centros urbanos en cada uno de los grupos o niveles jerárquicos establecidos inicialmente (Tabla 2).

42 Franz Gutiérrez Rey 
Tabla 1

Componentes, variables y clases para la determinación de la jerarquía urbana

\begin{tabular}{|c|c|c|}
\hline $\begin{array}{l}\text { COMPO- } \\
\text { NENTES }\end{array}$ & VARIABLES & CLASES \\
\hline \multirow{5}{*}{$\begin{array}{l}\text { I. } \\
\text { Administrativo }\end{array}$} & a. Investidura municipal & $\begin{array}{l}\text { Jerarquía administrativa: capital de } \\
\text { gobernación y cabecera municipal. }\end{array}$ \\
\hline & b. Ingresos municipales & Total de ingresos municipales. \\
\hline & c. Registro de instrumentos públicos & $\begin{array}{l}\text { Número de registros públicos: } \\
\text { documentos y certificados de tradición. }\end{array}$ \\
\hline & d. División notarial & $\begin{array}{l}\text { Clase de oficina: primera, segunda } \\
\text { o única. }\end{array}$ \\
\hline & e. División judicial & $\begin{array}{l}\text { Clase de despacho: sede de distrito, } \\
\text { circuito o juzgado. }\end{array}$ \\
\hline \multirow{5}{*}{$\begin{array}{c}\text { II. } \\
\text { Componente } \\
\text { de servicios y } \\
\text { equipamientos } \\
\text { públicos } \\
\text { básicos }\end{array}$} & a. Telecom. & $\begin{array}{l}\text { Número de abonados: primer nivel, } \\
\text { tercer nivel o no clasificado. }\end{array}$ \\
\hline & b. Administración regional Telecom. & Clase de oficina: nivel I, II, III o IV. \\
\hline & c. Sacrificio de ganado mayor & $\begin{array}{l}\text { Número degüellos de ganado mayor: } \\
\text { hembras y machos. }\end{array}$ \\
\hline & d. División regional de correo & $\begin{array}{l}\text { Clase de oficina: sede regional, oficina } \\
\text { principal, directa o indirecta. }\end{array}$ \\
\hline & e. Aeropuertos & Clase de aeropuerto: A, B, C, D, E-AF o G \\
\hline \multirow{6}{*}{$\begin{array}{c}\text { III. } \\
\text { Componente } \\
\text { de servicios y } \\
\text { equipamientos } \\
\text { comerciales } \\
\text { y bancarios }\end{array}$} & a. Cartera de bancos & Cartera neta en bancos. \\
\hline & b. Depósitos en bancos & Total depósitos en bancos. \\
\hline & c. Sucursales bancarias & Número de sucursales bancarias y agencias \\
\hline & d. Comercio mayoristas y minoristas & Número de establecimientos. \\
\hline & e. Capital de cooperativas & Patrimonio de cooperativas. \\
\hline & a. Educación superior & Número de alumnos. \\
\hline \multirow{6}{*}{$\begin{array}{c}\text { IV. } \\
\text { Componente } \\
\text { de servicios y } \\
\text { equipamientos } \\
\text { sociales }\end{array}$} & b. Educación básica y media & Número de alumnos. \\
\hline & c. Educación Sena & Número de alumnos atendidos. \\
\hline & d. Hospitales públicos & Número de camas. \\
\hline & e. Cobertura Instituto de Seguro Social & Número de afiliados. \\
\hline & f. Centros de atención de salud privados & Número de centros. \\
\hline & g. Organización de la salud & $\begin{array}{l}\text { Clase de atención: especializado, regional, } \\
\text { local, centro o puesto de salud. }\end{array}$ \\
\hline \multirow{7}{*}{$\begin{array}{c}\mathrm{V} . \\
\text { Componente } \\
\text { de servicios y } \\
\text { equipamientos } \\
\text { culturales, } \\
\text { religiosos } \\
\text { y medios } \\
\text { masivos de } \\
\text { comunicación }\end{array}$} & a. Bibliotecas & Número de libros para la consulta. \\
\hline & b. Museos & Número de establecimientos. \\
\hline & c. Salas de cine & Número de sillas o de salas. \\
\hline & d. Emisoras & Número de emisoras AM, FM o ambas. \\
\hline & e. Hoteles & Número de hoteles. \\
\hline & f. Prensa escrita & Número de periódicos distribuidos. \\
\hline & g. División eclesiástica & $\begin{array}{l}\text { Jerarquías: Diócesis-vicariato-prelatura, } \\
\text { vicaría, parroquia o temporal. }\end{array}$ \\
\hline
\end{tabular}




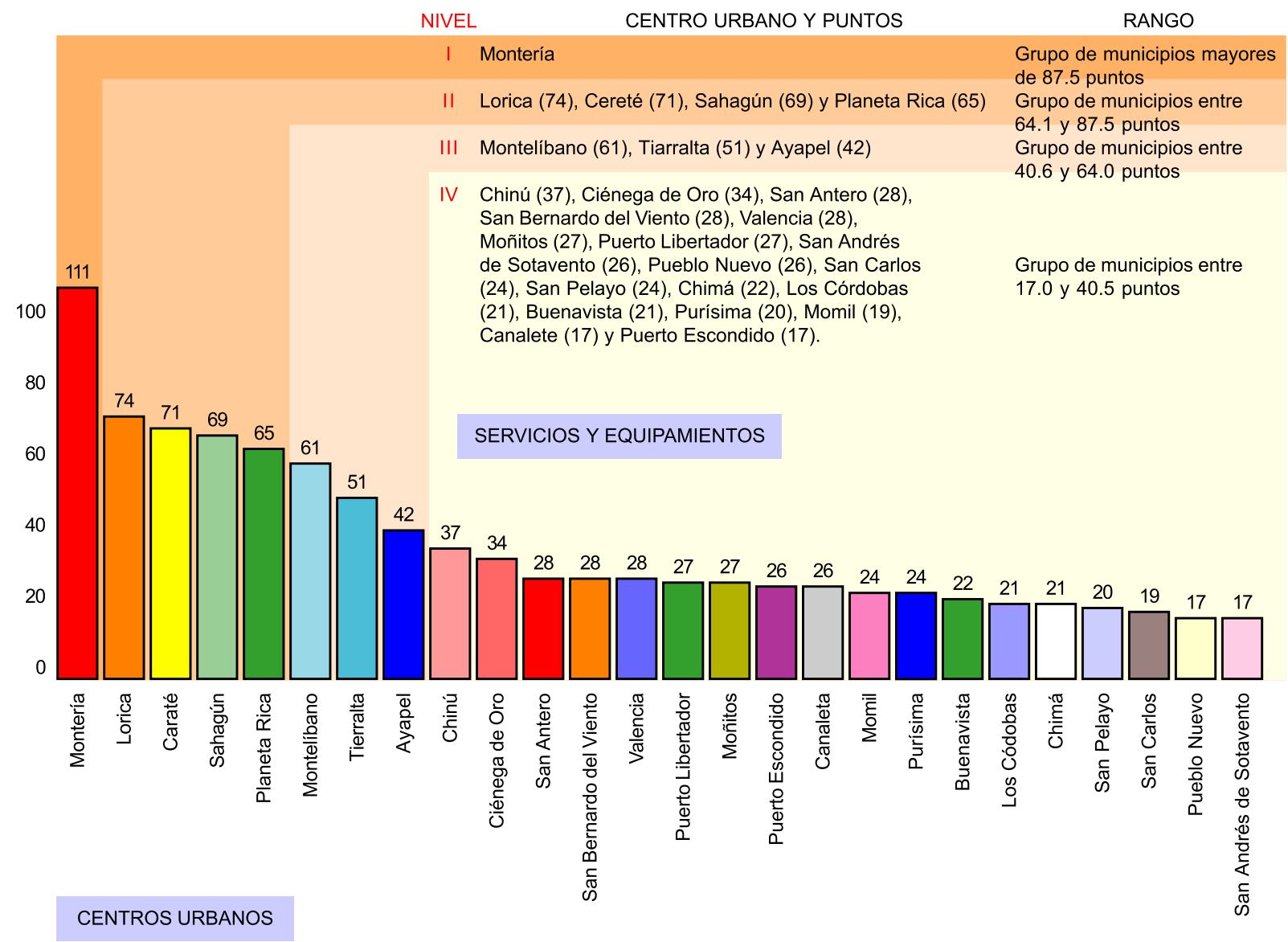

Figura 2. Niveles jerárquicos de los centros urbanos del departamento de Córdoba por primacía en la oferta de servicios y equipamientos. 
Tabla 2

Análisis de población para jerarquización urbana-Dinámica poblacionalcensos de 1985 y 1983

\begin{tabular}{|c|c|c|c|c|c|}
\hline $\begin{array}{l}\text { NIVEL } \\
\text { JERÁR- } \\
\text { QUICO }\end{array}$ & CENTRO URBANO & $\begin{array}{c}\text { CENSO } \\
1985\end{array}$ & DINÁMICA & CENTRO URBANO & $\begin{array}{c}\text { CENSO } \\
1993\end{array}$ \\
\hline I & Montería & 169.074 & $\rightarrow$ & Montería & 182.275 \\
\hline \multirow{5}{*}{ II } & Sahagún & 29.628 & $>$ & Sahagún & 33.648 \\
\hline & Planeta Rica & 27.249 & & Cereté & 31.657 \\
\hline & Cereté & 26.279 & & Planeta Rica & 31.391 \\
\hline & Lorica & 25.670 & & Montelíbano & 24.048 \\
\hline & Montelíbano & 21.243 & $\rightarrow$ & Lorica & 22.540 \\
\hline \multirow{7}{*}{ III } & Ciénaga de Oro & 15.102 & 7 & Tierralta & 19.595 \\
\hline & Ayapel & 14.075 & & Ciénaga de Oro & 18.008 \\
\hline & Chinú & 13.971 & & Ayapel & 15.792 \\
\hline & Tierralta & 13.695 & & Chinú & 14.265 \\
\hline & San Antero & 10.158 & & San Carlos & 13.050 \\
\hline & Móvil & 6.789 & & San Bernardo del Viento & 12.600 \\
\hline & Valencia & 6.700 & & Puerto Libertador & 12.240 \\
\hline \multirow{13}{*}{ IV } & San Bernardo del Viento & 5.940 & & Valencia & 7.846 \\
\hline & Pueblo Nuevo & 5.282 & & San Andrés de Sotavento & 6.958 \\
\hline & Purísima & 4.916 & & Móviel & 6.256 \\
\hline & Puerto Libertador & 4.113 & & Pueblo Nuevo & 5.755 \\
\hline & San Andrés de Sotavento & 3.979 & & San Antero & 5.244 \\
\hline & San Pelayo & 3.859 & & Purísima & 5.192 \\
\hline & Buenavista & 3.209 & & Buenavista & 3.578 \\
\hline & San Carlos & 3.104 & & Moñitos & 3.029 \\
\hline & Canalete & 3.103 & & Chimá & 1.741 \\
\hline & Moñitos & 2.789 & & Puerto Escondido & 1.717 \\
\hline & Chima & 2.249 & & San Pelayo & 1.671 \\
\hline & Puerto Escondido & 1.821 & & Canalete & 1.642 \\
\hline & Los Córdobas & 1.385 & $\rightarrow$ & Los Córdobas & 1.271 \\
\hline
\end{tabular}

Fuente: DANE, Censos de población 1985 y 1993. 
Tabla 3

Jerarquías urbanas establecidas para los centros del departamento de Córdoba a través de diferentes metodologías ${ }^{2}$

\begin{tabular}{|c|c|c|c|c|c|c|}
\hline \multirow{2}{*}{ 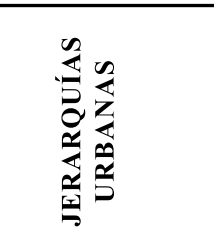 } & & \multicolumn{4}{|c|}{ ESTUDIOS, ESCALA Y AÑO } & \multirow{2}{*}{$\begin{array}{c}\text { SÍNTESIS } \\
\text { JERARQUÍA } \\
\text { URBANA } \\
\text { PROPUESTA } \\
\text { INICIAL DE LA } \\
\text { INVESTIGACIÓN }\end{array}$} \\
\hline & $\begin{array}{c}\text { Oferta de servicios } \\
\text { y equipamientos } \\
(2001) \\
\text { Departamental }\end{array}$ & $\begin{array}{l}\text { Análisis de tamaño } \\
\text { de población } \\
\text { (1993) } \\
\text { Departamental }\end{array}$ & $\begin{array}{c}\text { IGAC } \\
(1988-1996) \\
\text { Nacional }\end{array}$ & $\begin{array}{c}\text { Fundación } \\
\text { Social } \\
(1998) \\
\text { Nacional }\end{array}$ & $\begin{array}{c}\text { Plan de desarrollo } \\
\text { de Montería } \\
\text { (1991) } \\
\text { Departamental }\end{array}$ & \\
\hline $\begin{array}{l}\text { Primer nivel u } \\
\text { orden nacional }\end{array}$ & & Bogotá D. C. & Bogotá D. C. & Bogotá D. C. & Bogotá D. C. & Bogotá D. C. \\
\hline $\begin{array}{c}\text { Primer nivel } \\
\text { u orden } \\
\text { departamental }\end{array}$ & Montería & Montería & Montería & Montería & Montería & Montería \\
\hline $\begin{array}{c}\text { Segundo nivel } \\
\text { u orden } \\
\text { departamental }\end{array}$ & $\begin{array}{l}\text { Lorica } \\
\text { Cereté } \\
\text { Sahagún } \\
\text { Planeta Rica }\end{array}$ & $\begin{array}{l}\text { Lorica } \\
\text { Cereté } \\
\text { Sahagún } \\
\text { Planeta Rica } \\
\text { Montelíbano }\end{array}$ & $\begin{array}{l}\text { Lorica } \\
\text { Cereté } \\
\text { Sahagún } \\
\text { Planeta Rica }\end{array}$ & $\begin{array}{l}\text { Lorica } \\
\text { Cereté } \\
\text { Sahagún } \\
\text { Planeta Rica } \\
\text { Montelíbano }\end{array}$ & $\begin{array}{l}\text { Lorica } \\
\text { Cereté } \\
\text { Sahagún } \\
\text { Planeta Rica }\end{array}$ & $\begin{array}{l}\text { Lorica } \\
\text { Cereté } \\
\text { Sahagún } \\
\text { Planeta Rica } \\
\text { Montelíbano }\end{array}$ \\
\hline $\begin{array}{c}\text { Tercer nivel } \\
\text { u orden } \\
\text { departamental }\end{array}$ & $\begin{array}{l}\text { Montelibano } \\
\text { Tierralta } \\
\text { Ayapel }\end{array}$ & $\begin{array}{l}\text { Tierralta } \\
\text { Ayapel } \\
\text { Chinú } \\
\text { San Carlos } \\
\text { San Bernardo } \\
\text { Puerto Libertador }\end{array}$ & $\begin{array}{l}\text { Montelíbano } \\
\text { Tierralta } \\
\text { Ayapel } \\
\text { Chinú }\end{array}$ & $\begin{array}{l}\text { Tierralta } \\
\text { Ayapel } \\
\text { Chinú } \\
\text { Ciénaga de Oro }\end{array}$ & $\begin{array}{l}\text { Montelibano } \\
\text { Tierralta } \\
\text { Ayapel } \\
\text { Chinú }\end{array}$ & $\begin{array}{l}\text { Tierralta } \\
\text { Ayapel } \\
\text { Chinú }\end{array}$ \\
\hline Cuarto Nivel & $\begin{array}{l}\text { Chinú } \\
\text { Resto de municipios } \\
\text { (17) }\end{array}$ & $\begin{array}{l}\text { Resto de municipios } \\
\text { (14) }\end{array}$ & $\begin{array}{l}\text { Resto de munici- } \\
\text { pios (17) }\end{array}$ & $\begin{array}{l}\text { Resto de municipios } \\
\text { (16) }\end{array}$ & $\begin{array}{l}\text { Resto de municipios } \\
\text { (17) }\end{array}$ & $\begin{array}{l}\text { Resto de } \\
\text { municipios (17) }\end{array}$ \\
\hline
\end{tabular}

$\overline{{ }^{2} \text { Se utilizan }}$ diferentes procedimientos, pero sus indicadores son similares. 
Para Córdoba el resultado del análisis confirma la clasificación jerárquica por servicios y equipamientos e incorpora al municipio de Montelíbano al segundo nivel departamental por concentración de población -extracción de níquel en Cerromatoso-.

Los resultados obtenidos de la jerarquía urbana departamental por servicios y equipamientos y población urbana son posteriormente validados con otros estudios de nivel nacional y departamental (IGAC, 1986 y 1996; Fundación Social, 1998; Plan de Desarrollo de Montería, 1991) e integrados en una nueva síntesis matricial y para determinar compatibilidades o diferencias en los distintos niveles jerárquicos y proponer la jerarquía urbana -propuesta inicial de la investigación-, la que se va validando a través de los procesos de análisis posteriores -espaciales, de subregionalización y de subregiones funcionales- (Tabla 3 y Figura 1).

La propuesta de jerarquización urbana (columna 7, Tabla 3), es caracterizada a través de los análisis de la morfología, funciones y dinámicas del sistema urbano departamental -atributos-.

\subsection{Morfología del sistema urbano}

Toma dos atributos claves par el OTD: la distribución espacial y de tamaños.

La distribución espacial se desarrolla a través del índice de Clark-Evans (índice del elemento más próximo $-R_{n-1}$ ), para conocer el grado de concentración, dispersión, uniformidad o aleatoriedad de la distribución espacial de los centros urbanos del departamento: $R_{n}=2 * \bar{d} \sqrt{\frac{N}{S}}$, de donde, d: es la distancia promedio de cada asentamiento respecto al más próximo en un territorio de Superficie S, en el que existen $\mathrm{N}$ número de asentamientos.

Para el departamento de Córdoba este valor es de 1.3, lo que significa que la distribución espacial de los centros urbanos es de tipo aleatoria-radiocéntrica, hecho que está ligado a que la red vial y la administración del departamento son de tipo centralista (Mapa 1).

La distribución de tamaños se analiza por medio del índice de Primacía, el cual permite identificar la estructura de tamaños de los centros del departamento y su estado de equilibrio o desequilibrio:

$$
\begin{gathered}
\text { I p }=\frac{\text { P 1 }}{4} 100 \\
\sum_{1=1} \text { P 1 }
\end{gathered}
$$

De donde, $\mathrm{I}_{\mathrm{p}}$ es la población de la ciudad mayor del sistema $\left(\mathrm{P}_{1}\right)$, dividida entre la suma de la población de las cuatro ciudades mayores $\left(\mathrm{P}_{1}, \mathrm{P}_{2}, \mathrm{P}_{3}, \mathrm{P}_{4}\right)$ y multiplicada por 100. Su valor: 65.29 , expresa para el departamento de Córdoba que el sistema de asentamientos es de tipo macrocéfalo, es decir que existe una primera o única ciudad rectora - su capital Montería- y no existen dos o más ciudades que compitan por alcanzar la primacía departamental, originando desequilibrio en el sistema de centros urbanos del departamento ${ }^{3}$.

3 De acuerdo con la regla rango-tamaño, la población del segundo centro urbano Sahagún con 33.648 hab., debería ser la mitad de la ciudad mayor Montería (182.275 hab). Sahagún en población es tres veces menor a la ciudad de Montería, pero debería tener 91.000 hab. aproximadamente, por esto se infiere la existencia de desequilibrio poblacional que as $u$ vez se refleja en el nivel de oferta de servicios y equipamientos. 


\subsection{Funciones del sistema urbano}

El análisis se orienta a clasificar y caracterizar los centros a partir de sus funciones principales y se desarrolla por medio del método de "Nelson", que se apoya en el concepto de "empleo normal", es decir la proporción de empleo en cada una de las ramas de actividad económica. El resultado constituye el denominado "empleo normal" especialización en cada una de las ramas de actividad económica presentes en cada uno de los centros urbanos del departamento. Se considera que un centro está especializado en una determinada actividad cuando tiene una proporción de empleo en esa actividad mayor que la media, más la desviación típica (Tabla 4).

\section{Tabla 4}

Clasificación funcional de los centros urbanos del departamento de Córdoba por ramas de actividad económica

\begin{tabular}{|c|c|}
\hline CENTRO & ESPECIALIZACIÓN FUNCIONAL \\
\hline Montería & $\begin{array}{l}\text { Ramas VIII (comercio). IV (industria manufacturera).XV (servicios sociales y } \\
\text { salud). XI (administración pública). IX (transporte y almacenamiento). V (hoteles y } \\
\text { restaurantes). X (sector financiero). }\end{array}$ \\
\hline Ayapel & Ramas II (pesca). XV (servicios sociales y salud). \\
\hline Buenavista & $\begin{array}{l}\text { Ramas XIV (otras actividades comunitarias). XV (servicios sociales y salud). } \\
\text { Canalete Ramas I (agricultura y ganadería). XIV (otras actividades comunitarias). }\end{array}$ \\
\hline Cereté & Ramas IV (industria manufacturera). XV (servicios sociales y salud). X (sector financiero). \\
\hline Chimá & $\begin{array}{l}\text { Ramas III (explotación de minas). II (pesca). XII (actividad inmobiliaria). } \\
\text { XV (servicios sociales y salud). VI (electricidad. gas y agua). }\end{array}$ \\
\hline Chinú & $\begin{array}{l}\text { Ramas VIII (comercio). IV (industria manufacturera). IX (transporte y } \\
\text { almacenamiento). XV (servicios sociales y salud). }\end{array}$ \\
\hline Ciénaga de Oro & Ramas IV (industria manufacturera). XV (servicios sociales y salud). \\
\hline Lorica & Ramas VIII (comercio). XV (servicios sociales y salud). \\
\hline Los Córdobas & Ramas I (agricultura y ganadería). XII (actividades inmobiliarias). \\
\hline Momil & Ramas II (pesca). XV (servicios sociales y salud). \\
\hline Montelíbano & $\begin{array}{l}\text { Ramas III (explotación minas). VII (construcción). V (hoteles y restaurantes). Moñitos } \\
\text { Ramas IX (transporte y almacenamiento). X (sectorfinanciero). }\end{array}$ \\
\hline Planeta Rica & $\begin{array}{l}\text { Ramas VIII (comercio). IV (industria manufacturera). VII (construcción). } \\
\text { XV (servicios sociales y salud). XIV (otras actividades comunitarias). } \\
\text { V (hoteles y restaurantes). X (sector financiero). }\end{array}$ \\
\hline Pueblo Nuevo & Ramas VII (construcción). XV (servicios sociales y salud). X (sector financiero). \\
\hline Puerto Escondido & $\begin{array}{l}\text { Ramas I (agricultura y ganadería). XII (actividades inmobiliarias). } \\
\text { XV (servicios sociales y salud). }\end{array}$ \\
\hline Puerto Libertador & Ramas III (explotación minas). VI (electricidad. gas y agua). V (hoteles y restaurantes). \\
\hline Purísima & Ramas II (pesca). XV (servicios sociales y salud). \\
\hline Sahagún & $\begin{array}{l}\text { Ramas VIII (comercio). VI (construcción). XV (servicios sociales y salud). San Andrés de } \\
\text { Sotavento Ramas XIII (enseñanza). XII (actividades inmobiliarias). XV (servicios } \\
\text { sociales y salud). }\end{array}$ \\
\hline San Antero & Ramas I (agricultura y ganadería). V (hoteles y restaurantes). \\
\hline San Bernardo del Viento & $\begin{array}{l}\text { Ramas IX (transporte y almacenamiento). XV (servicios sociales y salud). San Carlos } \\
\text { Ramas I (agricultura y ganadería). XV (servicios sociales y salud). }\end{array}$ \\
\hline San Pelayo & $\begin{array}{l}\text { Ramas XIII (enseñanza). XIV (otras actividades comunitarias). } \\
\text { XV (servicios sociales y salud). }\end{array}$ \\
\hline Tierralta & Ramas VIII (comercio). XV (servicios sociales y salud). V (hoteles y restaurantes). \\
\hline Valencia & Ramas I (agricultura y ganadería). XV (servicios sociales y salud). \\
\hline
\end{tabular}

48 Franz Gutiérrez Rey 


\subsection{Dinámicas del sistema urbano}

El análisis de aspectos de las dinámicas urbanas como la metropolización, conurbación y suburbanización es muy importante para el OTD, pero obliga a abrir una ventana (escala), en la investigación para referenciar, evidenciar y mostrar los fenómenos que para el departamento de Córdoba aún no se hacen evidentes, a excepción de los niveles de urbanización. La metropolización, conurbación, suburbanización, reflejan un nivel de desarrollo superior o exagerado de algunos centros del sistema departamental -crecimiento físico y de población-advirtiendo a los planificadores sobre cambios drásticos en las necesidades de una población -demanda de servicios-.

Otro aspecto clave en los procesos de OTD, es el grado de urbanización por cuanto, permite conocer como están creciendo físicamente los centros urbanos. Este se obtiene por medio del índice de Pinchemel -medida que indica el grado de urbanización de manera simple y eficiente-: I = [(U2 / PR $) * 100]$, de donde $\mathrm{I}=$ Índice de urbanización, $\mathrm{U}=$ Población urbana de cada centro del departamento, $\mathrm{P}=$ Población total urbana del departamento y $\mathrm{R}=$ Población rural de cada municipio.

El índice en el contexto departamental permite determinar el valor que puede tener un centro urbano en relación con otro más importante, de tal manera que las cifras resultantes tienen un valor comparativo por el grado de urbanización. Éste índice de urbanización se analiza junto con el incremento de población ${ }^{4}$ en los últimos periodos inter-censales para cada uno de los centros urbanos. Los cuales deben ser directamente proporcionales para la planificación, es decir que a mayor incremento de población, debe existir un mayor valor en el número índice o grado de urbanización y viceversa (Tabla5).

\footnotetext{
4 Medidos por la razón $\left[\left(\left(P_{2}-P_{1}\right) / P_{1}\right) * 100\right]-$ donde $P_{2}=$ Censo 1993 y $P_{1}=$ Censo de 1985-, denominándose variación porcentual de la población en un municipio. Esta razón indica un grado de crecimiento pero no puede considerarse como tasa de crecimiento. Cabe anotar que las variaciones pueden ser positivas o negativas según aumente o disminuya la población.
} 
Tabla 5

Incrementos de la población e índice de urbanización -Porcentajes-

\begin{tabular}{|c|c|c|c|c|c|}
\hline CENTROS & $\begin{array}{c}\text { CENSO } \\
93\end{array}$ & $\begin{array}{c}\text { CENSO } \\
85\end{array}$ & $\begin{array}{l}\text { INCRE- } \\
\text { MENTO }\end{array}$ & CENTRO & $\begin{array}{c}\text { ÍNDICE } \\
\text { URBANI- } \\
\text { ZACIÓN }\end{array}$ \\
\hline San Carlos & 13.050 & 3.104 & 320.42 & Montería & 54.00 \\
\hline Puerto Libertador & 12.240 & 4.113 & 197.59 & Planeta Rica & 5.00 \\
\hline San Bernardo del Viento & 12.600 & 5.940 & 112.12 & PuertoLibertador & 5.00 \\
\hline San Andrés de Sotavento & 6.958 & 3.979 & 74.86 & Cereté & 3.00 \\
\hline Tierralta & 19.595 & 13.695 & 43.08 & Sahagún & 3.00 \\
\hline Cereté & 31.857 & 26.279 & 21.22 & Montelíbano & 3.00 \\
\hline Ciénaga de Oro & 18.006 & 15.102 & 19.22 & San Carlos & $\mathbf{\Lambda}_{2.00}$ \\
\hline Valencia & 7.846 & 6.700 & 17.10 & San Bernardo Viento & 2.00 \\
\hline Planeta Rica & 31.391 & 27.294 & 15.03 & Ciénaga de Oro & 2.00 \\
\hline Sahagún & 33.648 & 29.628 & 13.56 & Lorica / & 1.00 \\
\hline Montelíbano & 24.048 & 21.243 & 13.20 & Tierrala & 1.00 \\
\hline Ayapel & 15.792 & 14.075 & 12.19 & Ayapel & 1.00 \\
\hline Buenavista & 3.578 & 3.209 & 11.49 & Chytnú & 1.00 \\
\hline Pueblo Nuevo & 5.756 & 5.292 & 8.76 & Momil & 1.00 \\
\hline Moñitos & 3.029 & 2.798 & 8.57 & San Pelayo & 0.00 \\
\hline Montería & 182.275 & 169.074 & 7.80 & San Andrés & 0.00 \\
\hline Purísima & 5.192 & 4.916 & 5.61 & Purísima & 0.00 \\
\hline Chinú & 14.265 & 13.971 & 2.10 & Chimá & 0.00 \\
\hline Puerto Escondido & 1.717 & 1.821 & -5.71 & San Antero & 0.00 \\
\hline Los Córdobas & 1.271 & 1.365 & -6.88 & Moñitos & 0.00 \\
\hline Momil & 6.256 & 6.789 & -7.63 & Los Córdobas & 0.00 \\
\hline Lorica & 22.540 & 25.670 & -12.19 & Canalete & 0.00 \\
\hline Chimá & 1.741 & 2.249 & -22.58 & Puerto Escondido & 0.00 \\
\hline Canalete & 1.642 & 3.103 & -47.80 & Pueblo Nuevo & 0.00 \\
\hline San Antero & 5.244 & 10.158 & -48.37 & Buenavista & 0.00 \\
\hline San Pelayo & 1.671 & 3.856 & -56.66 & Valencia & 0.00 \\
\hline
\end{tabular}

Fuente: Censos DANE, 1985 y 1993.

\section{Los procesos espaciales}

Los análisis de jerarquía urbana y de los vínculos funcionales no deben tomarse por separado sino de manera complementaria, por esta razón continúa el diagnóstico del sistema urbano con fines de OTD, con el análisis de los vínculos administrativos $y$ socioeconómicos, ya que estos permiten identificar y definir las subregiones funcionales del departamento. 
Los vínculos administrativos son analizados por la distribución espacial de las instituciones del Estado en la prestación de servicios y los sociales y económicos, desde el punto de vista del movimiento de personas en la satisfacción de necesidades, junto con los de carácter fisico como el sistema vial departamental, los medios de transporte y la accesibilidad.

\subsection{Los vínculos administrativos}

Éste análisis para el OTD, permite identificar y espacializar a partir de los vínculos establecidos por la administración de instituciones del Estado, las diferentes subregionalizaciones generadas por el cubrimiento de la oferta y demanda de los servicios, para evaluar su funcionalidad - coherencia, armonía y eficacia espacial-.

El análisis de los vínculos administrativos, se funda en la teoría de que el Estado subregionaliza en un departamento áreas de acción administrativa a través de la ubicación y organización espacial de sus entidades o instituciones prestadoras de servicios y crea flujos de usuarios de carácter funcional o no.

Para desarrollar este análisis se identificaron en el departamento instituciones del orden nacional y/o departamental que cuentan con una organización administrativa receptora de flujos intermunicipales, cubrimiento departamental y estructuran áreas de influencia subregional. Por ejemplo: Superintendencia de Notariado y Registro, Despacho Judicial, Servicio de Salud, Servicio de Educación Pública, Policía Nacional y Oficina de Planeación Departamental. Cada una de las instituciones seleccionadas configura una matriz espacializable y compatible de la actuación del Estado a través de su ubicación y organización.

Este ejercicio permite establecer los centros urbanos nodos o polos de funciones administrativas por la oferta de servicios por parte de las entidades del Estado (Mapa 2); la oferta, demanda y desplazamientos $^{5}$ en la búsqueda de los servicios ofrecidos por estas instituciones ${ }^{6}$ (Mapa 3) y la síntesis de la centralidad de los servicios administrativos (Mapa 4).

El Mapa 4, presenta la compatibilización de las diferentes organizaciones administrativas existentes en el departamento y las optimiza desde el punto de vista funcional y de igual manera permite la verificación y complementación de los resultados obtenidos sobre la jerarquía urbana, desde el punto de vista de las subregionalización administrativa.

5 Los desplazamientos reflejan por sí mismos, el estado de integración o desintegración administrativa de las instituciones en el orden departamental -presencia del Estado como facilitador, centralizador de las relaciones y proveedor de servicios- y el avance de procesos de descentralización y organización administrativa.

6 El OTD, al revisar este tipo de situación en los departamentos contribuye a mejorar el equilibrio espacial organizativo y a lograr que el Estado tenga presencia positiva, eficiente y suficiente y no por el contrario, que ésta sea de tipo problemático o traumático para los usuarios. 


\subsection{Los vínculos socioeconómicos}

El análisis se desarrolla a través del estudio de los flujos, frecuencias e intensidad del transporte intermunicipal, los cuales facilitan las relaciones socioeconómicas y permiten la identificación de las áreas o subregiones de relación económica y social en el departamento, permitiendo corroborar la jerarquía urbana establecida por los análisis de servicios y equipamientos, población y vínculos administrativos. Para esto, se parte de la teoría de que las personas para satisfacer sus necesidades están sujetas a la relación de ofertademanda de los servicios de transporte y a la condición del sistema vial y estos, a su vez determinan el nivel de acceso a los servicios y equipamientos -tiempo y distancia- ${ }^{7}$.

El análisis de los vínculos socioeconómicos, recopila y actualiza la información sobre la red vial-vías por entidad de conservación, clasificación técnica, estado y distancias-, el sistema de transporte -interdepartamental e intermunicipal y volúmenes de tránsito- y el movimiento de pasajeros flujos--. Los datos especializados permiten la construcción del mapa de áreas o subregiones de integración socioeconómica (Mapa 5).
Identificada, caracterizada y espacializada la red vial, los vínculos de transporte y flujos de pasajeros, al igual que los volúmenes de tránsito o flujo vehicular, se realiza una nueva síntesis -integración matricial y espacial- de las áreas o subregiones de integración socioeconómica, identificándose para el departamento los nodos de relación social y económica.

\subsection{Análisis de accesibilidad}

Los mínimos tiempos empleados y las menores distancias recorridas para la satisfacción de necesidades por las personas del departamento -facilidad de llegar a un centro-, son consecuencia directa de una mejor accesibilidad e integración subregional y departamental que se deriva de las condiciones de la red vial, es decir que a mayor accesibilidad mayor integración departamental y subregional y viceversa. El análisis de la accesibilidad intermunicipal establece la funcionalidad de las subregiones identificadas anteriormente por los vínculos administrativos y socioeconómicos y se realiza cubriendo dos aspectos: la accesibilidad de los centros urbanos del departamento a la capital y de los centros nodos de subregión a los municipios polarizados.

7 El autor estima que el transporte de pasajeros en un $80 \%$ representa las relaciones comerciales con base en necesidades a satisfacer, es decir que la mayoría de las personas se movilizan en función económica o comercial (trabajo, búsqueda de empleo, cobranzas, compra y ventas de mercancías, entre otros) y que el $20 \%$ restante lo hacen por relaciones de tipo social (visitas a familiares, amistades, asistencia a eventos culturales, estudio o recreación).

52 Franz Gutiérrez Rey 


\section{Los procesos de subregionalización}

Los actores departamentales tienen diferentes propuestas de subregiones y puntos de vista. Algunas subregionalizaciones propuestas cuentan con la aceptación de grupos de personas, las cuales las promueven por razones o intereses propios de su competencia.

\subsection{Subregiones propuestas por actores departamentales}

Estas subregiones propuestas en y para el departamento, se compatibilizan -proceso de integración basado en la similitud espacial de las áreas propuestas-, con las subregiones de integración administrativa y socioeconómica-resultados anteriores de la investigación-.

Ejemplos de propuestas de subregionalización en el departamento de Córdoba: por poblamiento, Unidad Regional de Planificación Agropecuaria -URPA-, Unidades Desconcentradas de Apoyo Municipal -UDAM-, Plan Nacional de Rehabilitación -PNR-, Gran Urabá, Corporación Autónoma Regional de los Valles del Sinú y San Jorge -CVS-, Folclóricas y Gobierno Departamental.

\subsection{Compatibilización}

La integración espacial tiene implícita la armonización de intereses para la planificación del territorio (Mapa 6). En este mapa, por ejemplo: las líneas de mayor grosor -rojas- en los límites de las subregiones representan la convergencia o compatibilidad espacial del $100 \%$ de los intereses expresados en las diferentes propuestas; las líneas de espesor medio -carmelitas- significan una convergencia espacial de la mayoría de intereses o propuestas (mayor del $50 \%$ ) y las líneas delgadas -naranja-, significan la convergencia de pocos intereses o de propuestas de subregionalización (menor al 50\%).

\subsection{Asociaciones de municipios}

Además de las propuestas de subregionalización departamentales, existen en la mayoría de los departamentos, asociaciones de municipios de tipo temporal, permanente, voluntarias u obligatorias, las cuales, se consideran unidades supramunicipales de integración político-territorial.

Éstas asociaciones de municipios son creadas por razones políticas o intereses económicos locales, conformando "subregiones" que permiten en su análisis espacial, conocer los procesos de integración territorial -cohesión-y definir la solidez de las subregiones propuestas para el OTD - concreción-, es decir que por medio del análisis espacial de las asociaciones municipales, se pueden validar las subregiones propuestas para el OTD (Mapa 7). Un ejemplo aplicado a lo anterior del departamento de Córdoba son las asociaciones de los municipios del Caribe -ASOMCARIBE-, Ciénaga Grande del Bajo Sinú -ASOMUCIBAS-, San Jorge y Alto Sinú y Urabá Norteño -AMASUN-. 


\subsection{Unidades territoriales}

Validadas las subregiones propuestas a través de las asociaciones municipales (Mapa 7), se procede a realizar el análisis espacial sobre la distribución de las unidades territoriales municipales que integran el departamento, en cuanto a su forma-límites-, extensión-superficie-y segregación -separación-. La primera se da cuando algunos municipios no permiten el desarrollo de otros, en razón de su forma limítrofe; la segunda, por que no controlan su territorio debido a su extensión o superficie y la tercera por que a pesar de que se erigen como municipios -creación, siguen dependiendo del que se sustrajeron.

Ejemplos de este tipo de disfuncionalidad espacial en el departamento se encuentran por forma, la que se presenta entre los municipios de Montelíbano y Puerto Libertador; por segregación, la del municipio de Canalete al separarse de los Córdobas y por extensión, el municipio de Tierralta.

\section{Subregiones funcionales}

Las subregiones funcionales establecidas por la jerarquía urbana, el tamaño de la población, la administración de entidades departamentales, las áreas de influencia socioeconómica, las propuestas de subregionalización existentes, la red vial, las condiciones de accesibilidad y las asociaciones municipales, son una base sólida, valida y suficiente para la propuesta de las subregiones funcionales y de la jerarquía urbana del departamento (Mapa 7).

\subsection{Evaluación}

La propuesta de subregiones funcionales es evaluada a través de la identificación de sus oportunidades, limitantes y problemas - OLP-, de acuerdo con el método general para el OTD, establecido por el IGAC en 1977 (Figura 3).

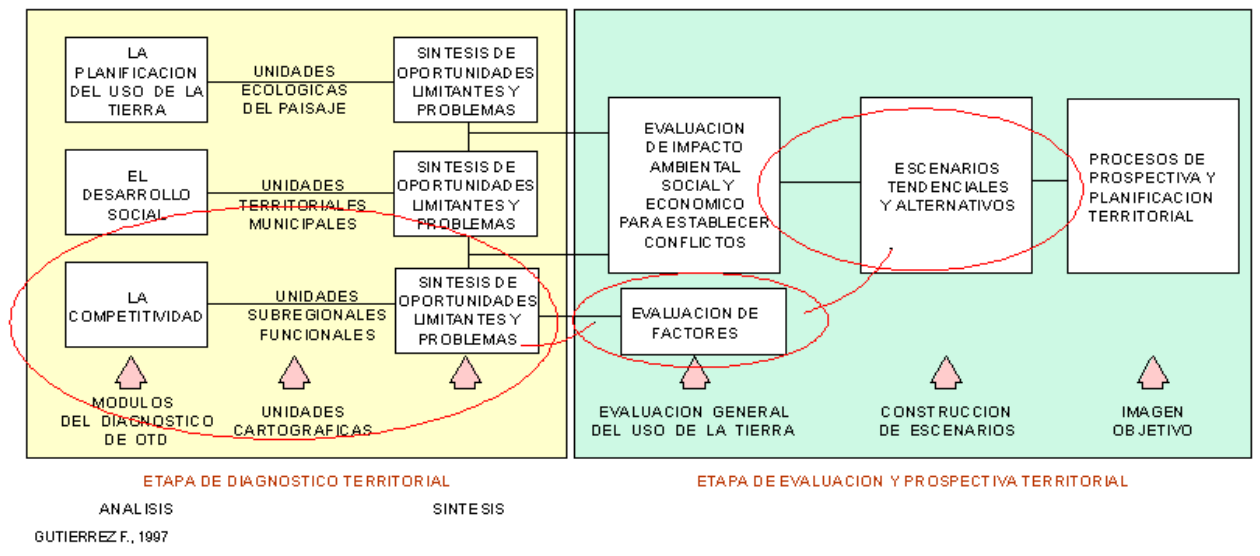

Figura 3. Etapas del proceso ordenador.

54 Franz Gutiérrez Rey 
La matriz de oportunidades, limitantes y problemas -OLP-, es fundamental para plantear los procesos o acciones de planificación, en la búsqueda del equilibrio espacial del sistema urbano departamental y de la organización de los centros urbanos, en beneficio de los habitantes del departamento (Tabla 6 y Mapa 8).

El valor de un proceso evaluativo y prospectivo aplicado a la organización funcional del sistema urbano, con fines de OTD, está en la superación objetiva de la visión del corto plazo y su labor es la de orientar y organizar la reflexión colectiva para la construcción de futuro (Figura 4). La investigación no desarrolla el proceso prospectivo, en razón de su complejidad; pero toma de éste el análisis de los planes y programas de desarrollo futuro, para examinar la viabilidad de las subregiones funcionales planteadas (Mapa 7).

La prospectiva territorial para los componentes de OTD, tiene en cuenta los hechos portadores de futuro, es decir en este caso, los que implican una afectación leve o fuerte de la organización del sistema urbano actual y su tendencia-futuro-, como los proyectos de desarrollo sobre la red vial, la conformación de nuevas unidades territoriales -creación de nuevos municipios-y los acuerdos en la solución de conflictos sociales -creación de nuevas entidades territoriales, por ejemplo: indígenas-.

\subsection{Síntesis}

Con base en los análisis y síntesis anteriores, se construye el mapa de subregiones funcionales del departamento de Córdoba para el POTD -imagen objetivo- y se estructura la jerarquía urbana para esta propuesta, junto con las acciones que deben emprenderse en el caso de aceptación (Mapa 8, Figura 5 y Tabla 6), en el marco general de la evaluación de los factores de la competitividad departamental, el componente social y de la planificación del uso de la tierra -PUT-, para el OTD (Figura 3).

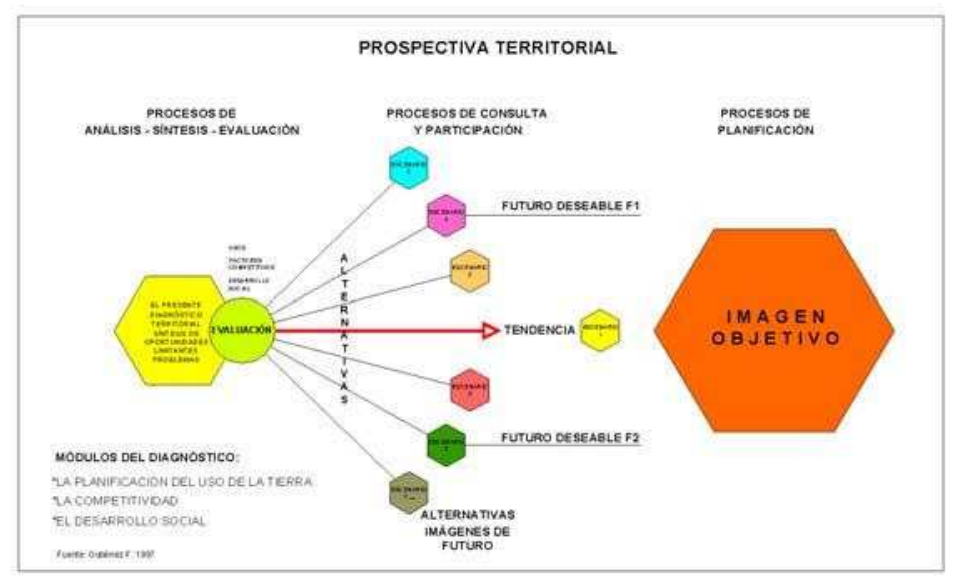

Figura 4. Prospectiva territorial. 
Oportunidades, limitantes y problemas de la propuesta de subregionalización funcional

\begin{tabular}{|c|c|c|c|c|}
\hline \begin{tabular}{|c|} 
CENTRO \\
SUBREGIÓN \\
\end{tabular} & $\begin{array}{c}\text { CENTROS } \\
\text { POLARIZADOS } \\
\end{array}$ & OPORTUNIDADES & LIMITANTES & PROBLEMAS \\
\hline LORICA & $\begin{array}{l}\text { Moñitos } \\
\text { San Bernardo del } \\
\text { Viento } \\
\text { San Antero } \\
\text { Purísima } \\
\text { Momil } \\
\text { Chimá }\end{array}$ & $\begin{array}{l}\text { - Adecuada oferta de servicios y equipamientos en Lorica } \\
\text { para su jerarquía y centros urbanos demandantes. } \\
\text { - Área de integración administrativa. } \\
\text { - Área de integración socioeconómica. } \\
\text { - Adecuada accesibilidad e integración espacial de los } \\
\text { centros urbanos de Momil, Purísima y San Antero. } \\
\text { - Adecuada condición de la red vial -pavimentada- para } \\
\text { los centros urbanos de Purísima, Momil y San Antero. } \\
\text { - Área propuesta por todos los actores } \\
\text { departamentales. } \\
\text { - Área propuesta por la investigación. }\end{array}$ & $\begin{array}{l}\text { - Moderada condición de la red } \\
\text { vial de acceso a los centros } \\
\text { urbanos de San Bernardo del } \\
\text { Viento, Moñitos y Chimá (vías } \\
\text { secundarias regionales sin pa- } \\
\text { vimentar). }\end{array}$ & $\begin{array}{l}\text { - Accesibilidad e integra- } \\
\text { ción espacial con los } \\
\text { centros urbanos de San } \\
\text { Bernardo del Viento y } \\
\text { Moñitos. } \\
\text { - Integración en planes y } \\
\text { programas de los centros } \\
\text { urbanos costeros de San } \\
\text { - Antero, San Bernardo del } \\
\text { Viento y Moñitos respec- } \\
\text { to a Purísima, Momil y } \\
\text { Chimá. } \\
\end{array}$ \\
\hline CERETÉ & $\begin{array}{l}\text { Ciénaga de Oro } \\
\text { San Pelayo } \\
\text { San Carlos }\end{array}$ & $\begin{array}{l}\text { - Área de integración administrativa. } \\
\text { - Área de integración socioeconómica. } \\
\text { - Adecuada accesibilidad e integración } \\
\text { - espacial de los centros urbanos } \\
\text { - Adecuada condición de la red vial (pavimentada). } \\
\text { - Área propuesta por todos los actores } \\
\text { departamentales. } \\
\text { - Área propuesta por la investigación }\end{array}$ & $\begin{array}{l}\text { - Moderada oferta de servicios y } \\
\text { equipamientos en el centro } \\
\text { urbano nodal de Cereté para su } \\
\text { jerarquía. }\end{array}$ & \\
\hline
\end{tabular}



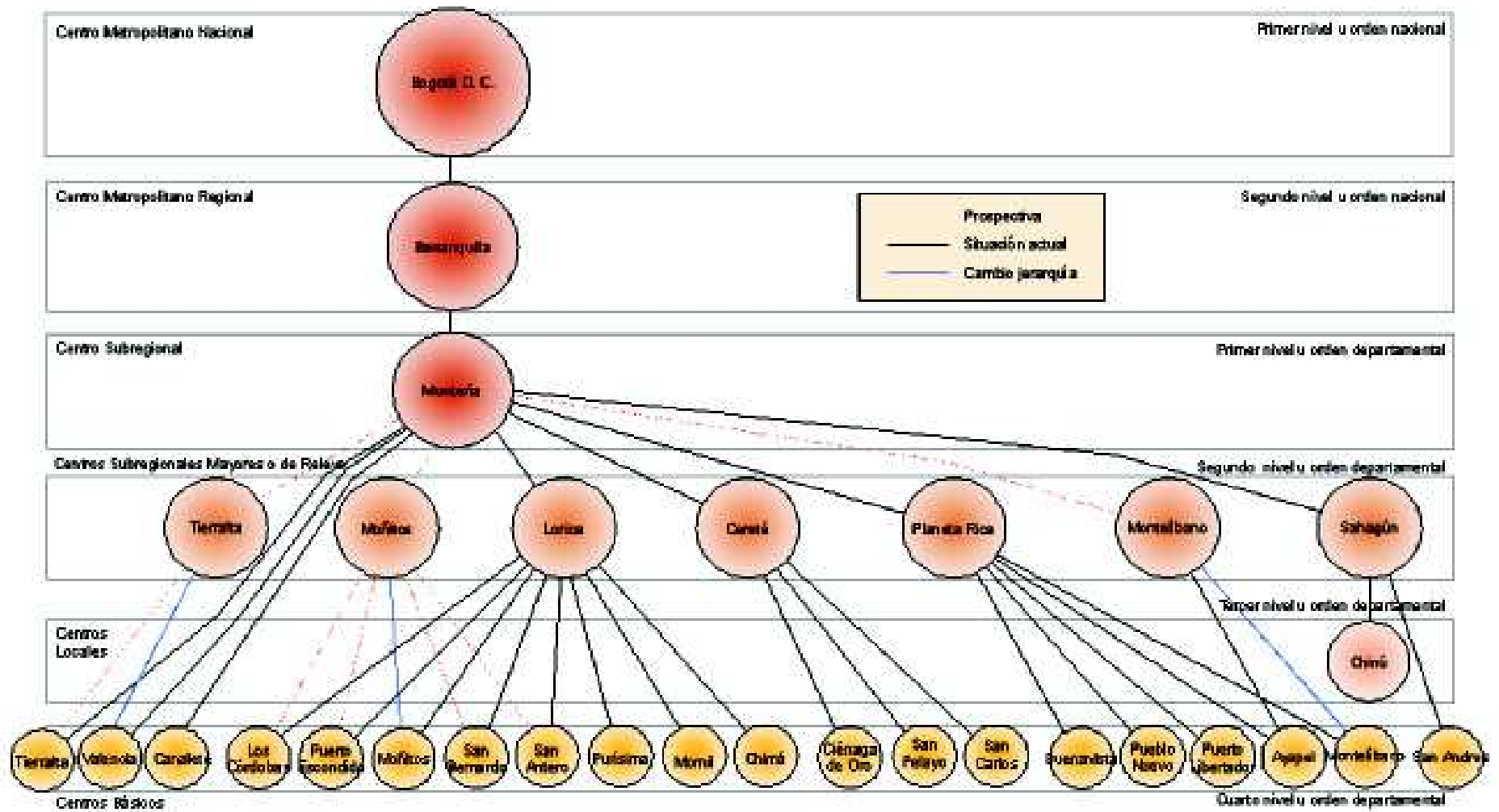

Figura 5. Propuesta para una distribución geográfica equilibrada del desarrollo: prospección del ordenamiento de la funcionalidad. 


\section{Bibliografía}

Fundación Social. Municipios y regiones de Colombia. Una mirada desde la Sociedad Civil. Editorial Antropos. Bogotá D. C., 1998.

Gobernación de Córdoba. Plan de desarrollo del municipio de Montería. El sistema urbano regional del departamento de Córdoba, Departamento Nacional de Planeación-DNP-Consejo Regional de Planificación de la Costa Atlántica-CORPES- Corporación Autónoma de los Valles del Sinú y San Jorge-CVS-, Montería, 1991.

Gutiérrez, Franz. Caquetá dinámicas de un proceso, Reordenamiento administrativo y auge urbano. Capítulo IX, Instituto Amazónico de Investigaciones Científicas-SINCHI-, Ministerio del Medio Ambiente, Tercer Mundo Editores, Colombia, 2000; pp. 55-73.

Diseño metodológico para el diagnóstico de la organización funcional del sistema urbano en los planes de ordenamiento territorial departamental: el caso de Córdoba. Tesis de Maestría. Convenio Universidad Pedagógica y Tecnológica de Colombia-UPTC-e Instituto Geográfico Agustín Codazzi-IGAC-, Escuela de Posgrado en Geografía-EPG-, Bogotá D. C., 2001.

La organización e integración funcional del sistema urbano en el departamento del Putumayo. Proyecto de Caracterización de los Asentamientos Humanos. Instituto Amazónico de Investigaciones Científicas-SINCHI-, Bogotá, 1999.

La organización e integración funcional del sistema urbano en el departamento de Caquetá. Instituto Amazónico de Investigaciones Científicas -SINCHI-, Proyecto de caracterización de los asentamientos humanos de los departamentos del Caquetá y Putumayo: Dinámicas y perspectivas, la dimensión espacio funcional, Bogotá D. C., 1999.

Propuesta de ordenamiento territorial para la zona rural del municipio de Ibagué. Zonificación rural. Alcaldía de Ibagué, Instituto Geográfico Agustín Codazzi-IGAC-, Sociedad Colombiana de Arquitectos-SCA-, Regional Tolima, 1999.

58 Franz Gutiérrez Rey 
Gutiérrez, Franz. Fase de formulación del POTD, etapas de evaluación y prospectiva territorial. Sin publicar, Proyecto Piloto de Apoyo a los Procesos de Ordenamiento y Desarrollo territorial, Instituto Geográfico Agustín Codazzi-IGAC-Subdirección de Geografía, 1997.

Gutiérrez Franz et al. Caquetá construcción de un territorio amazónico en el Siglo XX, Sistema urbano. Capítulo 5, Instituto Amazónico de Investigaciones Científicas-SINCHI-, Ministerio del Medio Ambiente, Tercer Mundo Editores, Colombia, diciembre 2000; pp. 167 a 161.

Instituto Geográfico Agustín Codazzi -IGAC-. Bases conceptuales y metodológicas para la formulación del plan de ordenamiento territorial departamental. Editorial Linotipia Bolívar, Bogotá D.C., 1997.

Atlas de Colombia, CD-ROOM, Bogotá D. C., 1996.

Estructura urbano regional de Colombia, Bogotá D.E., 1986.

Montañéz, Gustavo y Domínguez, Camilo. Aportes de la geografía a la regionalización. En: Revista Colombia, Ciencia y tecnología, Universidad Nacional de Colombia, Departamento de Geografía, Bogotá D. C.: Vol. 10, No. 4, 1993.

Negrete, Víctor. En Busca del Desarrollo. Conclusiones y recomendaciones, Seminario sobre ordenamiento y autonomía territorial en el departamento de Córdoba, Montería, 28 y 29 de octubre de 1992.

Rincón, Hugo. Organización espacial y funcional del municipio de Florencia-Caquetá-. Tesis de Maestría. Convenio Universidad Pedagógica y Tecnológica de Colombia-UPTC-e Instituto Geográfico Agustín Codazzi -IGAC-, Estudio de Posgrado en Geografía -EPG--, Bogotá D. C., 2001.

Sánchez, Olga. Organización espacial del municipio de Facatativa como centro de relevo: una mirada a su región funcional. Tesis de Maestría. Convenio Universidad Pedagógica y Tecnológica de Colombia-UPTC-e Instituto Geográfico Agustín Codazzi-IGAC-, Estudio de Posgrado en Geografía-EPG-, Bogotá D. C., 2001. 
